\title{
Correction to: A Reduced Hardware ISI and Mismatch Shaping DEM Decoder
}

\author{
Vincent O'Brien $^{1}$ (D) Anthony G. Scanlan ${ }^{1}{ }_{(\mathbb{D})} \cdot$ \\ Brendan Mullane ${ }^{1}$ (D)
}

\section{Correction to: Circuits Syst Signal Process https://doi.org/10.1007/s00034-017-0681-8}

The original version of the article unfortunately contained an error in figure. The presentation of Fig. 2 is incorrect. Figure 1 was erroneously duplicated and published as Fig. 2 in the article. The corrected Fig. 2 is given below:

The original article can be found online at https://doi.org/10.1007/s00034-017-0681-8.

$\triangle$ Vincent O’Brien

vincent.obrien@ul.ie

Anthony G. Scanlan

tony.scanlan@ul.ie

Brendan Mullane

brendan.mullane@ul.ie

1 University of Limerick, Limerick, Ireland 


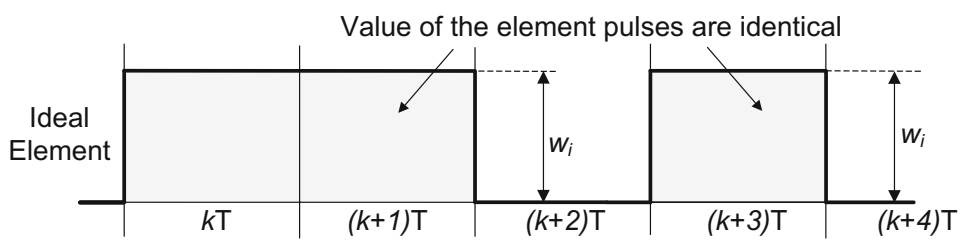

(a)

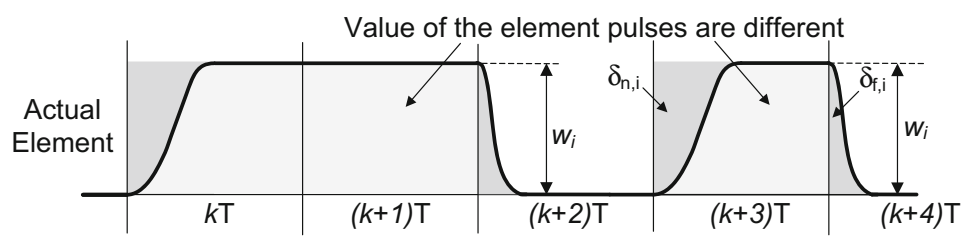

(b)

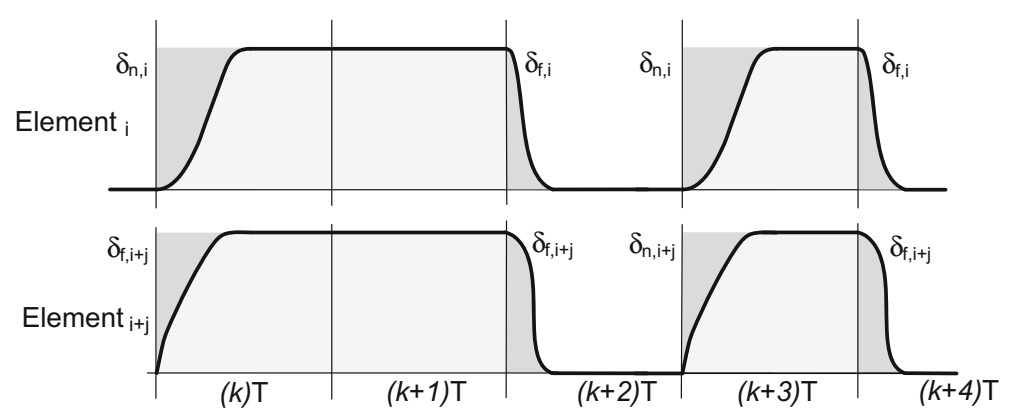

(c)

Fig. 2 a Ideal pulse, $\mathbf{b}$ actual pulse $\delta_{\mathrm{n}, \mathrm{i}} \neq \delta_{\mathrm{f}, \mathrm{i}}$, $\mathbf{c}$ variation in rise and fall errors between elements $\delta_{\mathrm{n}, \mathrm{i}} \neq \delta_{\mathrm{n}, \mathrm{i}+\mathrm{j}} \delta_{\mathrm{f}, \mathrm{i}} \neq \delta_{\mathrm{f}, \mathrm{i}+\mathrm{j}}$ 